\title{
Motor content norms for 4,565 verbs in Spanish
}

\author{
Romina A. San Miguel Abella ${ }^{1}$ - María González-Nosti ${ }^{1}$
}

Published online: 12 April 2019

(C) The Psychonomic Society, Inc. 2019

\begin{abstract}
Embodiment theory suggests that, during the processing of words related to movement, as in the case of action verbs, somatotopic activation is produced in the motor and premotor cortices. In the same way, some studies have demonstrated that patients with frontal-lobe damage, such as Parkinson's patients, have difficulties processing that kind of stimulus. At the moment, no standardized data exist concerning the motor content of Spanish verbs. Therefore, the aim of the present research was to develop a database of 4,565 verbs in Spanish through a survey filled out by 152 university students. The value for the motor content was obtained by calculating the average value from the answers of the participants. In addition, the reliability of the results was estimated, as well as their convergent validity, using diverse correlation coefficients. The database and the raw responses of the participants can be downloaded from this website: https://inco.grupos.uniovi.es/enlaces.
\end{abstract}

Keywords Motor content $\cdot$ Verbs $\cdot$ Database $\cdot$ Norms

Over the years, great advances in the study of language processing have allowed the discovery of a dissociation at the brain level between the centers responsible for processing nouns and verbs. Pioneering work in this area was performed by Damasio and Tranel (1993), who designed a task of naming nouns and verbs. This task was performed by two groups of patients, one with lesions in the middle temporal cortex, and the other with damage in the left frontal cortex. The results showed a double dissociation between both groups. The patients with frontal damage had difficulties to recover the verbs but not the nouns, whereas those with temporal damage found nouns challenging, but not verbs.

Subsequent studies have confirmed the existence of this dissociation in disorders such as aphasia (Druks, 2002), progressive nuclear palsy (Cotelli et al., 2006), corticobasal degeneration (Cotelli et al., 2006; Silveri \& Ciccarelli, 2007) and motor neuron disorder (Bak \& Hodges, 2004).

In this same line, Vigliocco, Vinson, Druks, Barber, and Cappa (2011) conducted a review study of the existing

Electronic supplementary material The online version of this article (https://doi.org/10.3758/s13428-019-01241-1) contains supplementary material, which is available to authorized users.

María González-Nosti

gonzaleznmaria@uniovi.es

1 Department of Psychology, University of Oviedo, Oviedo, Spain literature to date on whether word processing of different grammatical classes (especially nouns and verbs) involves different neural systems. They found that some studies showed a confusion between semantic (objects vs. actions) and grammatical distinctions (nouns vs. verbs), and also found a further confusion between studies related to simple wordprocessing mechanisms and sentence integration. In all of these studies, there was a clear neuronal distinction between the processing of words that refer to objects (nouns) and action words (typical verbs). In addition, the studies also concluded that the grammatical effects of the type of word emerged or became stronger for tasks and languages that impose greater processing demands. This indicates that grammar classes alone would not explain the organization of brain knowledge. Semantics (meaning) and pragmatics (usage) should also be considered, as well as the distributive keys of language related to the syntactic behavior of verbs and nouns in sentences (e.g., position in sentences) and to their morphosyntactic marking (e.g., types of inflection in nouns vs. verbs), which distinguishes between nouns and verbs. In recent decades, the incorporation of neuroimaging techniques, such as functional magnetic resonance (fMRI), to the study of language has been able to specify with greater accuracy the brain locus where words are processed at the semantic level.

When applying fMRI in healthy participants, it has been discovered that the same areas involved in the realization of movement or the reception of sensations are involved in the processing of the language related to those movements and sensations. This is what is 
described by embodiment theory. This theory explains that semantic content that makes reference to a movement is composed of information that is represented within the motor and sensory systems (Mahon \& Caramazza, 2008). Likewise, Hauk, Johnsrude, and Pulvermüller (2004), demonstrated that the activation produced at the cerebral level by the processing words referring to actions performed with the arm and the leg (e.g., catching or kicking) corresponded to the activation that occurs when these movements were performed effectively. These results have been supported by numerous studies that have shown that there really is a concordance between the frontal regions involved in the processing of action words and the motor areas that allow realization of the actions to which reference is made. That is to say, the motor and premotor cortices, besides being involved in the planning and execution of movements, are also active during the processing of words semantically related to those same movements (Aziz-Zadeh, Wilson, Rizzolatti, \& Iacoboni, 2006; Boulenger, Hauk, \& Pulvermüller, 2009; Fogassi, Gallese, \& Rizzolatti, 2002).

Perceptual and bodily experiences are both indispensable to form the semantic component of actions, since they provide the knowledge about their importance as components of the meaning of words. This type of experience will activate sensory-motor states when external contextual stimuli are received, and also when thinking about them (Barsalou, 1999, 2008; Paivio, 1991). This principle means that in order for action word processing to be produced, the activation of previously learned mental schemata is required. These schemas must be formed with perceptual and motor information of the parts of the body involved in the movement associated with a specific concept, such as an action verb.

Bien, Jost, Khader, Mertens, and Rösler (2009) developed a study with healthy participants, with the intention of specifying more the cerebral localization of the processing of verbs. The task the participants had to execute was to subvocally complete a sentence formed by two nouns, generating a verb for it (e.g., "vampire-blood," so that the participant completes with the verb "to suck"). The strongest activation for the generation of verbs was found in the lower left prefrontal cortex and the upper left temporal gyrus. The latter area is involved in the perception of movement. These results are further evidence that the perceptual representations of movements mediate the generation of verbs. Another study has shown that not only are the literal meanings of such verbs processed in motor areas, but even figurative meanings that refer to different parts of the body produce similar activations (Boulenger et al., 2009). The task posed by Boulenger et al. was to present literal phrases and English idioms related to both the hand (e.g., "John got the object" and "John got the idea") and the leg (e.g.,
"Pablo kicked the ball" and "Pablo kicked the habit," which means "stop doing something that is hard to stop doing"). They verified that the activity patterns depended fundamentally on the part of the body (arm or leg) to which the word referred. The regions of motor and premotor cortex were activated in a stronger way than other areas during the reading of these sentences, observing a common network in cortical activity for both conditions.

However, in the study of Brass, Friederici, and Rüschemeyer (2007), the researchers showed that not all verbs activate the motor regions of the brain. These authors compared the activation of action verbs and abstract verbs in German. In the case of action verbs, reference is made to those that have a specific motor meaning (e.g., running), whereas verbs of the abstract or sensory type are those that do not contain a motor-type meaning (e.g., thinking or listening). The results showed greater activation in the somatosensory and motor cortex, but only for action verbs. This, for the authors, suggested the existence of a functional relationship between the lexical processing of action verbs and the sensorimotor system, which differs from the lexical processing of verbs with abstract meaning. Other researchers, such as de Zubicaray, Arciuli, and McMahon (2013), on the contrary, interpreted the motor cortex activity as a reflection of the implicit processing of ortho-phonological statistical regularities that help to distinguish the grammatical class of a word. Likewise, other studies, such as that of Rodríguez-Ferreiro, Gennari, Davies, and Cuetos (2011), considered that differential brain activation related to action and abstract verbs could be due to the greater demands on semantic recovery or integration of properties imposed by abstract verbs. In recent years, some authors have proposed a softened version of embodiment, based on the results of some patients who, despite showing sensorimotor impairments, hardly present difficulties in conceptual processing. This "disembodied" position suggests that a certain concept can be distributed through specific systems of multiple modalities, so that a reduction in sensorimotor information would cause the processing of a concept dependent on the specific information of the modality to rely more on other types of representations (Mahon \& Caramazza, 2008; Mahon \& Hickok, 2016).

Anyway, on the basis of these findings, some researchers have raised the possibility that patients with damage in the frontal lobe experience difficulties in processing action verbs.

This is the case for Parkinson's disease (PD), a degenerative and chronic disorder of the nervous systemcaused by deterioration of the basal ganglia and characterized by difficulties in the initiation and integration of 
movements (Hornykiewicz \& Kish, 1984)—which translates into motor symptoms such as hypokinesia, bradykinesia, and limited length and amplitude of repetitive movements of the extremities (Bloxham, Dick, \& Moore, 1987; Miller Koop, Hill, \& Bronte-Stewart, 2013). In addition, language is one of the cognitive processes that is affected in patients with PD, since the deterioration can spread to frontostriatal areas that are important to language functioning. The dysfunction in semantic processing, due to the subcortical frontal deficit, causes words that are related to an action to be processed with greater difficulty than those that are not. Thus, in a study by Fernandino et al. (2013), a lexical decision task was designed in which half of the verbs referred to voluntary actions of the hand and arm (e.g., gripping, pressing), and the other half were related to abstract concepts (e.g., believing, improving). The results showed that, in relation to the controls, the patients with PD had processing of action verbs that was more impaired than the processing of abstract verbs, showing higher error rates and longer reaction times. Another study, carried out by Herrera, Rodríguez-Ferreiro, and Cuetos (2012), showed that, in addition, these patients showed special difficulty with action verbs that have a high degree of motor content, as compared to those with low levels. The task that was carried out was the naming of drawings that implied some kind of action. The actions were classified according to the motor component of the verb to which they referred (high motor componente.g., running - vs. low motor component-e.g., sewing). The results obtained showed that the difficulty presented by these patients was due to the semantic nature of the verbs, since there was an effect of the level of movement content that the verbs generated.

One of the most widespread treatments for PD is Levodopa, a precursor of dopamine that replaces endogenous dopamine, in which the patient is deficient. In addition to improving motor symptoms, it has also been found to have a beneficial effect on cognitive symptoms. Cuetos and Herrera (2013) kept in mind the effect of this medication when developing one of their studies. When performing a verbal fluency task, patients suffering from $\mathrm{PD}$ with and without medication (ON and OFF, respectively) were compared, and also compared with healthy participants. The task presented a specific number of nouns and verbs orally. The participants had to say as quickly as possible the first word that came to their minds once they had heard each of the stimuli. When the patients were not under the effect of Levodopa (OFF medication), they generated words with less strength of association with the word provided, for both verbs and nouns, in comparison with the participants to whom the medication had been supplied (ON medication). Interestingly, the patients with PD without medication produced words with less strength of association than among the healthy controls only in response to verbal stimuli. Therefore, this is an indication that the absence of dopamine affects the correct functioning of the semantic lexical system, dispersing the activation to semantically unrelated words, especially with verbal stimuli.

Finally, in one of the most recent studies, Herrera, Bermúdez-Margaretto, Ribacoba, and Cuetos (2015) used a task based on the verbal fluency of actions. In the results, they found that patients without medication generated a lower number of verbs of high motor specificity, and that this number increased significantly with the administration of dopamine to these same participants. Verbs of high specificity are those that refer to a specific part of the body necessary to perform an action (e.g., writing), whereas verbs with low motor specificity do not imply a single determined part of the body (e.g., swimming).

Although most studies of difficulties in processing motor verbs have been conducted with PD patients, recent research has extended the focus to other frontal-lobe injury disorders, such as amyotrophic lateral sclerosis (ALS). Thus, Cousins, Ash, and Grossman (2018) compared a group of PD patients with ALS patients who might have high or low motor impairment in a spontaneous narrative task. The results showed a dissociation in the processing of action verbs in ALS high-motor-impairment patients, since processing was affected only for body verbs (those in which the body was the agent of the action; e.g., grab), but not for theme verbs (those in which the body receives the action; e.g., fall). This finding was related to decreased gray matter volume in premotor cortex. Such a dissociation was not present in the group of PD patients, whose achievement was low with all action verbs.

Bayram, Muhittin, and Akbostanci (2018), on the other hand, compared the performance in a verb fluency task of a group of patients with PD, a hypokinetic movement disorder, and of another group of patients with primary cervical dystonia (PCD), a hyperkinetic movement disorder characterized by involuntary movements and abnormal positions of the head and neck, due to dysfunctions in the brain stem, spinal cord, and motor cortex (mainly basal ganglia and cerebellum). Both groups produced fewer action verbs than the control group. It is important to continue doing research and studies to further define the types of cognitive alterations that patients with frontal-lobe damage suffer from, but for that, more tools are needed. Despite the large number of works carried out related to the processing of verbs, we know of no database up to the present time documenting the motor component of Spanish verbs, nor, as far as we know, has any been developed in other languages. 
Until now, the procedure that has been used has generated a new database every time a study has been completed, using different samples of participants and collecting data under different conditions. In many cases, the results obtained in the databases generated by the different studies have not been comparable.

Therefore, we have produced a database of the motor component of thousands of verbs in use in Spanish, as a means to encourage future research, thus favoring more precise comparisons between studies. In addition, since the norms refer to a semantic quality of verbs, we consider it plausible that the present database will be useful for research developed in other languages, since the conceptual properties of most of the verbs in the database are likely to remain constant.

\section{Method}

\section{Participants}

One hundred fifty-two university students, selected by a nonprobabilistic convenience-sampling technique, took part in this experiment. Each questionnaire was responded to by between 23 and 29 participants, with 25 participants being the average for the 52 questionnaires. Spanish was a requirement for all participants, so the response lines of participants whose native language was not Spanish had to be eliminated. Almost all the student respondents were studying for a degree in psychology at the University of Oviedo. The average age of the participants who completed the questionnaires was 22 years old.

The participants who responded to the questionnaires were $84.6 \%$ women and $15.4 \%$ men (for a detailed description of the sample, see Appendix Table 3). Each person completed between one and ten questionnaires.

\section{Materials}

Preparation of the questionnaires that were handed out to the participants started with a database of 4,640 verbs in Spanish (Alonso, Díez, \& Fernandez, 2016). Of all the verbs, a total of 4,565 were selected from the initial database; that is, 75 verbs were excluded because they were low-frequency words in Spanish, such as ademar, concomitar, or melgar, and participants would probably not know their meaning.

The selected verbs were randomly distributed and assigned to each of the 52 questionnaires that made up the study. Subsequently, they were distributed among the different participants. The questionnaires were created using an extension of the "Google Drive" platform called "Google Forms," directly connected to the Google cloud.
The questionnaires were composed of 100 verbs or items, and these items were evaluated on a scale of scores from 1 to 7. The lowest scores corresponded to verbs that, in the opinion of the participants, implied very little movement (e.g., to vegetate), and the highest scores were linked to verbs that involved a lot of movement (e.g., to train). The participants could use the different scores to adjust their responses so they were as accurate as possible.

\section{Procedure}

Each questionnaire was sent to the same number of students of each course for the psychology and speech therapy degrees at the University of Oviedo, by email.

After a few days, those who did not have a sufficient number of answers (a minimum of 20) were sent to other students of different university degrees.

In the emails, potential participants were informed that the questionnaire was a completely anonymous test in which there were no correct or incorrect answers.

The instructions received by the participants for the completion of each questionnaire were as follows:

The next questionnaire aims to measure the degree of the motor component of the verbs that appear below. Your task is to assess, on a scale of 1 to 7 , the amount of mobility that each of these actions entails. Understanding as mobility, the amount of displacement and/or movement of the different parts of the body (fingers, hands, legs, etc.) involved in its realization. You must indicate with a 1 the verbs with a low motor component (e.g., to reason), and with a 7 those that have a high motor component (e.g., to plough).

In each questionnaire, the example verbs were varied so that they did not coincide with the words included as items, since they could have an influence in the scores of the participants. The assessment of the example words was carried out in other questionnaires.

All the items had to be answered obligatorily, and the participants were allowed to use the dictionary if they were unsure of the meaning of any one of them. Once the questionnaire was finished, participants were to send their answers by clicking on the Send button. The scores provided by all the participants for each item were collected in an Excel spreadsheet created by the program. Each questionnaire was rated by between 23 and 29 participants. The raw responses of the participants, as well as the complete database, can be consulted and downloaded from the website http://inco.grupos.uniovi.es/enlaces. In addition, the averages and standard deviations for each verb are provided in Appendix B. 
Data collection from the questionnaires was carried out between February 2016 and December 2017.

\section{Results}

The average (motor component grade), standard deviations, and the upper and lower range of the scores of the 4,565 verbs were calculated from the database.

To verify that there were no significant differences between the ages of the 52 groups of participants, a univariate variance analysis test was performed. Age was the dependent variable, and questionnaire was a fixed effect. The results obtained were not significant $(p=1)$, which indicates that the ages of the participants did not differ between groups.

With regard to the gender of the participants, the average percentage of female participants $(84.6 \%)$ was much higher than that of male participants (15.4\%). A maximum of 25 and a minimum of 16 women responded to each questionnaire $(S D=2.18)$. It was not necessary to eliminate the data of any participant, according to the variability criterion that was used. The maximum and minimum of the total of the scores given to the verbs by each participant were calculated, and in all there was variation.

For the analysis of the scores awarded to each verb, the intraclass correlation coefficient (ICC) was applied. This is considered the most appropriate way to quantify the reliability between different measurements associated with numerical variables. The results of this statistic oscillate between 0 and 1 , with a value of 1 referring to the maximum possible agreement between the scores, and a value of 0 making reference to no agreement. The advantage of this test is that it avoids dependency of the order of the correlation coefficient, since it estimates the average of the correlations among all the possible orderings of the pairs of observations. In contrast, the correlation coefficient only quantifies the linear association between two scores, but not the degree of agreement between them. One must bear in mind that variability can be due to two components: differences between the different participants, and differences between the measurements of each participant. The ICC is the proportion of the total variability that is due to the variability between participants (Pita-Fernández, Pértega-Díaz, \& Rodríguez Maseda, 2003).

This index was calculated for each of the different questionnaires, along with Cronbach's alpha, because it is interesting to check that there is agreement between the scores assigned by all the participants who responded to the same item.
Table 1 Measures of reliability of the questionnaires

\begin{tabular}{|c|c|c|c|}
\hline Questionnaire & Average Measures ICC & Sig. (ICC) & Cronbach's Alpha \\
\hline 1 & .949 & .001 & .959 \\
\hline 2 & .934 & .001 & .953 \\
\hline 3 & .943 & .001 & .957 \\
\hline 4 & .937 & .001 & .956 \\
\hline 5 & .943 & .001 & .962 \\
\hline 6 & .916 & .001 & .946 \\
\hline 7 & .936 & .001 & .961 \\
\hline 8 & .903 & .001 & .931 \\
\hline 9 & .911 & .001 & .937 \\
\hline 10 & .924 & .001 & .949 \\
\hline 11 & .930 & .001 & .949 \\
\hline 12 & .932 & .001 & .952 \\
\hline 13 & .901 & .001 & .935 \\
\hline 14 & .906 & .001 & .937 \\
\hline 15 & .916 & .001 & .941 \\
\hline 16 & .951 & .001 & .961 \\
\hline 17 & .910 & .001 & .936 \\
\hline 18 & .917 & .001 & .945 \\
\hline 19 & .922 & .001 & .945 \\
\hline 20 & .916 & .001 & .943 \\
\hline 21 & .910 & .001 & .937 \\
\hline 22 & .903 & .001 & .928 \\
\hline 23 & .930 & .001 & .949 \\
\hline 24 & .937 & .001 & .954 \\
\hline 25 & .917 & .001 & .941 \\
\hline 26 & .875 & .001 & .921 \\
\hline 27 & .941 & .001 & .941 \\
\hline 28 & .912 & .001 & .937 \\
\hline 29 & .914 & .001 & .947 \\
\hline 30 & .883 & .001 & .936 \\
\hline 31 & .886 & .001 & .935 \\
\hline 32 & .904 & .001 & .954 \\
\hline 33 & .887 & .001 & .945 \\
\hline 34 & .888 & .001 & .947 \\
\hline 35 & .922 & .001 & .943 \\
\hline 36 & .933 & .001 & .952 \\
\hline 37 & .963 & .001 & .971 \\
\hline 38 & .963 & .001 & .977 \\
\hline 39 & .934 & .001 & .958 \\
\hline 40 & .944 & .001 & .964 \\
\hline 41 & .921 & .001 & .952 \\
\hline 42 & .950 & .001 & .966 \\
\hline 43 & .942 & .001 & .958 \\
\hline 44 & .948 & .001 & .964 \\
\hline 45 & .948 & .001 & .964 \\
\hline 46 & .943 & .001 & .959 \\
\hline 47 & .958 & .001 & .968 \\
\hline 48 & .960 & .001 & .969 \\
\hline 49 & .962 & .001 & .970 \\
\hline
\end{tabular}


Table 1 (continued)

\begin{tabular}{llll}
\hline Questionnaire & Average Measures ICC & Sig. (ICC) & Cronbach's Alpha \\
\hline 50 & .958 & .001 & 969 \\
51 & .961 & .001 & 971 \\
52 & .959 & .001 & 968 \\
\hline
\end{tabular}

Average measures of a random-effect model for a factor in which the effects of people are random.

According to assessment of the ICCs, in this case there was a very good correspondence $(>.90)$ between the scores of the participants (see Table 1).

Doing this same test for the total of the 4,565 verbs jointly, we also obtained a high concordance between the participants for each item $(\mathrm{ICC}=.903, p<.001)$.

To establish the concurrent validity of the present work, and because, to date, as far as we know, there has been no complete database to evaluate this variable, we compared the results to data collected by other researchers for a work already published (Herrera \& Cuetos, 2012).

For this purpose, a selection of 50 verbs was made that coincided with those included in this work (see the comparison in Table 2), and a simple linear correlation was made, obtaining a Pearson's $r$ value of .864 , which shows that there was a high positive correlation.

The final database, which can be consulted and downloaded from the website http://inco.grupos.uniovi. es/enlaces, has been completed with age-of-acquisition (AoA) data taken from Alonso et al. (2016), as well as with data on frequency, logarithm of frequency, length in letters and syllables, orthographic and phonological neighborhood, familiarity, imageability, and concreteness, all taken from the EsPal database (Duchon, Perea, Sebastián-Gallés, Martí, \& Carreiras, 2013).

\section{Discussion}

During the last few years, several investigations have demonstrated differences in the processing of verbs and nouns in

Table 2 Values of the 50 common stimuli used to measure concurrent validity

\begin{tabular}{lllll}
\hline & Average & $S D$ & Min & Max \\
\hline Herrera \& Cuetos (2012) & 3.62 & 1.75 & 1.14 & 6.86 \\
Present study & 3.55 & 1.37 & 1.49 & 6.44 \\
\hline
\end{tabular}

both healthy people (Hauk et al., 2004) and patients with different disorders (Bak \& Hodges, 2004; Cotelli et al., 2006; Damasio \& Tranel, 1993; Druks, 2002; Silveri \& Ciccarelli, 2007).

These studies suggest that the frontal lobe, specifically the motor regions, may be involved in the processing of actions, especially those whose meaning implies a high degree of movement (Brass et al., 2007). Some explanations, such as the embodiment theory, maintain that semantic information related to movement in some verbs is represented within the motor and sensory systems. Other authors (Mahon \& Caramazza, 2008; Mahon \& Hickok, $2016)$ have defended a "softer" version, the "disembodiment" theory, which accepts that information relating to one verb may be distributed through specific systems of multiple modalities, including the motor system, but that a reduction in sensorimotor information would mean that the processing will rely more on information of other modalities.

In any case, research carried out in patients with Parkinson's disease, amyotrophic lateral sclerosis and primary cervical dystonia shows difficulties in these people to process verbs with a high motor component (Bayram et al., 2018; Cousins et al., 2018; Fernandino et al., 2013; Herrera \& Cuetos, 2012) which, in the case of PD, improve with medication (Cuetos \& Herrera, 2013; Herrera et al., 2015).

However, despite all the available research on the processing of motor and abstract verbs, until now there has not been a single database in any language that would allow for querying and unifying data about this semantic property of actions.

The norm set associated with this work provides subjective data on the motor component for a total of 4,565 verbs in Spanish. The study was carried out via surveys filled out by 152 university students. The value of the motor content was obtained by calculating average scores from the responses of the participants. At a statistical level, it demonstrated a high reliability calculated using the ICC, which allows the degree of agreement between the scores of different participants to be calculated, as well as in terms of Cronbach's alpha. Convergent validity, also appropriate, was obtained by a correlation analysis with the motor component values obtained from the study of Herrera and Cuetos (2012). These features make the database a useful tool for research.

The availability of these norms is crucial, because they provide standardized values for this variable, avoiding the need to use time to collect these data and allowing for the replication of results, which will provide great possibilities for the investigation of language in patients with PD or other disorders that cause damage to the frontal lobe (e.g., fronto-temporal dementia). 


\section{Appendix A}

Table 3 Number, gender, and age of participants in the 52 questionnaires

\begin{tabular}{|c|c|c|c|c|c|c|c|c|c|}
\hline \multirow[b]{2}{*}{ Questionnaire } & \multirow[t]{2}{*}{ Number of Participants } & \multicolumn{4}{|c|}{ Gender } & \multicolumn{4}{|l|}{ Age } \\
\hline & & Men & $\%$ Men & Women & $\%$ Women & Average & $S D$ & Max. & Min. \\
\hline 1 & 26 & 4 & 15 & 22 & 85 & 22.04 & 2.03 & 29 & 19 \\
\hline 2 & 26 & 5 & 19 & 21 & 81 & 21.92 & 1.90 & 29 & 20 \\
\hline 3 & 26 & 6 & 23 & 20 & 77 & 22.42 & 4.86 & 45 & 19 \\
\hline 4 & 28 & 7 & 25 & 21 & 75 & 22.61 & 5.13 & 45 & 18 \\
\hline 5 & 27 & 7 & 26 & 20 & 74 & 21.78 & 5.15 & 45 & 18 \\
\hline 6 & 27 & 8 & 30 & 19 & 70 & 22.22 & 5.02 & 45 & 18 \\
\hline 7 & 27 & 3 & 11 & 24 & 89 & 22.59 & 5.20 & 45 & 18 \\
\hline 8 & 29 & 4 & 14 & 25 & 86 & 22.83 & 4.92 & 45 & 19 \\
\hline 9 & 26 & 4 & 15 & 22 & 85 & 22.35 & 2.43 & 29 & 18 \\
\hline 10 & 26 & 2 & 08 & 24 & 92 & 21.96 & 2.46 & 29 & 19 \\
\hline 11 & 26 & 3 & 12 & 23 & 88 & 22.19 & 3.91 & 40 & 20 \\
\hline 12 & 27 & 5 & 19 & 22 & 81 & 21.67 & 2.76 & 35 & 20 \\
\hline 13 & 26 & 3 & 12 & 23 & 88 & 22.35 & 3.90 & 40 & 20 \\
\hline 14 & 26 & 5 & 19 & 21 & 81 & 21.67 & 2.76 & 35 & 20 \\
\hline 15 & 26 & 3 & 12 & 23 & 88 & 22.46 & 3.93 & 40 & 20 \\
\hline 16 & 25 & 4 & 16 & 21 & 84 & 22.35 & 4.60 & 40 & 20 \\
\hline 17 & 27 & 2 & 07 & 25 & 93 & 21.70 & 1.88 & 28 & 20 \\
\hline 18 & 27 & 5 & 19 & 22 & 81 & 21.52 & 2.74 & 35 & 20 \\
\hline 19 & 26 & 2 & 08 & 24 & 92 & 21.54 & 1.45 & 27 & 19 \\
\hline 20 & 25 & 3 & 12 & 22 & 88 & 21.77 & 3.05 & 35 & 20 \\
\hline 21 & 26 & 2 & 08 & 24 & 92 & 21.81 & 1.88 & 28 & 20 \\
\hline 22 & 26 & 4 & 15 & 22 & 85 & 21.77 & 3.05 & 35 & 20 \\
\hline 23 & 26 & 5 & 19 & 21 & 81 & 21.81 & 1.83 & 28 & 20 \\
\hline 24 & 26 & 5 & 19 & 21 & 81 & 21.65 & 1.72 & 28 & 20 \\
\hline 25 & 27 & 5 & 19 & 22 & 81 & 21.78 & 1.80 & 28 & 20 \\
\hline 26 & 26 & 5 & 19 & 21 & 81 & 21.65 & 1.72 & 28 & 20 \\
\hline 27 & 26 & 5 & 19 & 21 & 81 & 21.65 & 1.72 & 28 & 20 \\
\hline 28 & 26 & 5 & 19 & 21 & 81 & 21.81 & 1.83 & 28 & 20 \\
\hline 29 & 26 & 2 & 08 & 24 & 92 & 22.00 & 2.45 & 32 & 20 \\
\hline 30 & 25 & 2 & 08 & 23 & 92 & 22.00 & 2.50 & 32 & 20 \\
\hline 31 & 26 & 2 & 08 & 24 & 92 & 21.96 & 2.46 & 32 & 20 \\
\hline 32 & 26 & 2 & 08 & 24 & 92 & 21.96 & 2.46 & 32 & 20 \\
\hline 33 & 26 & 2 & 08 & 24 & 92 & 21.85 & 2.38 & 32 & 20 \\
\hline 34 & 26 & 2 & 08 & 24 & 92 & 21.85 & 2.38 & 32 & 20 \\
\hline 35 & 24 & 2 & 08 & 22 & 92 & 22.33 & 4.17 & 40 & 20 \\
\hline 36 & 24 & 2 & 08 & 22 & 92 & 22.33 & 4.17 & 40 & 20 \\
\hline 37 & 24 & 1 & 04 & 23 & 96 & 22.17 & 3.05 & 30 & 19 \\
\hline 38 & 23 & 2 & 09 & 21 & 91 & 22.17 & 3.14 & 30 & 19 \\
\hline 39 & 24 & 3 & 13 & 21 & 88 & 22.33 & 2.82 & 30 & 19 \\
\hline 40 & 23 & 3 & 13 & 20 & 87 & 22.22 & 2.76 & 30 & 19 \\
\hline 41 & 24 & 3 & 13 & 21 & 88 & 21.71 & 2.68 & 30 & 19 \\
\hline 42 & 23 & 3 & 13 & 20 & 87 & 21.74 & 2.80 & 30 & 19 \\
\hline 43 & 23 & 4 & 17 & 19 & 83 & 23.04 & 4.25 & 37 & 19 \\
\hline 44 & 23 & 4 & 17 & 19 & 83 & 23.04 & 4.16 & 37 & 19 \\
\hline 45 & 23 & 5 & 22 & 18 & 78 & 22.52 & 2.74 & 30 & 19 \\
\hline 46 & 23 & 5 & 22 & 18 & 78 & 22.52 & 2.74 & 30 & 19 \\
\hline 47 & 23 & 6 & 26 & 17 & 74 & 22.26 & 3.22 & 33 & 19 \\
\hline 48 & 23 & 5 & 22 & 18 & 78 & 21.74 & 2.22 & 28 & 19 \\
\hline 49 & 23 & 4 & 17 & 19 & 83 & 21.78 & 3.90 & 37 & 19 \\
\hline 50 & 23 & 5 & 22 & 18 & 78 & 22.26 & 4.55 & 37 & 19 \\
\hline 51 & 23 & 5 & 22 & 18 & 78 & 22.00 & 3.84 & 37 & 19 \\
\hline 52 & 23 & 7 & 30 & 16 & 70 & 22.00 & 3.88 & 37 & 19 \\
\hline Average & 25.23 & 3.88 & 15.4 & 21.35 & 84.6 & 22.07 & 3.1 & & \\
\hline
\end{tabular}

SD: Standard deviation; Max: Maximum; Min: Minimum. 


\section{References}

Alonso, M. A., Díez, E., \& Fernandez, A. (2016). Subjective age-ofacquisition norms for 4,640 verbs in Spanish. Behavior Research Methods, 48, 1337-1342. doi:https://doi.org/10.3758/s13428-0150675-Z

Aziz-Zadeh, L., Wilson, S. M., Rizzolatti, G., \& Iacoboni, M. (2006). Congruent embodied representations for visually presented actions and linguistic phrases describing actions. Current Biology, 16, 1818-1823. doi:https://doi.org/10.1016/j.cub.2006.07.060

Bak, T. H., \& Hodges, J. R. (2004). The effects of motor neurone disease on language: Further evidence. Brain and Language, 89, 354-361.

Barsalou, L. W. (1999). Perceptual symbol systems. Behavioral and Brain Sciences, 22, 577-609, disc. 609-660. doi:https://doi.org/10. 1017/S0140525X99002149

Barsalou, L. W. (2008). Cognitive and neural contributions to understanding the conceptual system. Current Directions in Psychological Science, 17, 91-95.

Bayram, E., Muhittin, C., \& Akbostanci, C. (2018). Verb naming fluency in hypokinetic and hyperkinetic movement disorders. Cortex, 100, 21-31.

Bien, S., Jost, K., Khader, P. H., Mertens, M., \& Rösler, F. (2009). Neural correlates of generating visual nouns and motor verbs in a minimal phrase context. Brain Research, 8, 122-132.

Bloxham, C. A., Dick, D. J., \& Moore, M. (1987). Reaction times and attention in Parkinson's disease. Journal of Neurology, Neurosurgery, and Psychiatry, 50, 11978-11983.

Boulenger, V., Hauk, O., \& Pulvermüller, F. (2009). Grasping ideas with the motor system: Semantic somatotopy in idiom comprehension. Cerebral Cortex, 19, 1905-1914.

Brass, M., Friederici, A. S., \& Rüschemeyer, S. A. (2007). Comprehending prehending: Neural correlates of processing verbs with motor stems. Journal of Cognitive Neuroscience, 19, 855-865.

Cotelli, M., Borroni, B., Manenti, R., Alberici, A., Calabria, M., Agosti, C., . . . Cappa, S. F. (2006). Action and object naming in frontotemporal dementia, progressive supranuclear palsy, and corticobasal degeneration. Neuropsychology, 20, 558-565.

Cousins, K. A. Q., Ash, S., \& Grossman, M. (2018). Production of verbs related to body movement in amyotrophic lateral sclerosis (ALS) and Parkinson's Disease (PD). Cortex, 100, 127-139.

Cuetos, F., \& Herrera, E. (2013). Semantic disturbance for verbs in Parkinson's disease patients off medication. Journal of Neurolinguistics, 26, 737-744.

Damasio, A., \& Tranel, D. (1993). Nouns and verbs are retrieved with differently distributed neural systems. Proceedings of the National Academy of Sciences, 90, 4957-4960.

de Zubicaray, G., Arciuli, J., \& McMahon, K. (2013). Putting an "end" to the motor cortex representations of action words. Journal of Cognitive Neuroscience, 25, 1957-1974. doi:https://doi.org/10. 1162/jocn_a_00437

Druks, J. (2002). Verbs and nouns-A review of the literature. Journal of Neurolinguistics, 15, 289-315.

Duchon, A., Perea, M., Sebastián-Gallés, N., Martí, A., \& Carreiras, M. (2013) EsPal: One-stop Shopping for Spanish Word Properties. Behavior Research Methods, 45, 1246-1258. doi:https://doi.org/ 10.3758/s13428-013-0326-1

Fernandino, L., Conant, L., Binder, J., Blindauer, K., Hiner, B., Spangler, K., \& Desai, R. (2013). Parkinson's disease disrupts both automatic and controlled processing of action verbs. Brain and Language, 127, $65-74$.

Fogassi, L., Gallese, V., \& Rizzolatti, G. (2002). Motor and cognitive functions of the ventral. Current Opinion in Neurobiology, 12, $149-154$.

Hauk, O., Johnsrude, I., \& Pulvermüller, F. (2004). Somatotopic representation of action words in human motor and premotor cortex. Neuron, 41, 301-307. doi:https://doi.org/10.1016/S0896-6273(03) 00838-9

Herrera, E., Bermúdez-Margaretto, B., Ribacoba, R., \& Cuetos, F. (2015). The motor-semantic meanings of verbs generated by Parkinson's disease patients on/off dopamine medication in a verbal fluency task. Journal of Neurolinguistics, 36, 72-78.

Herrera, E., \& Cuetos, F. (2012). Action naming in Parkinson's disease patients on/off dopamine. Neuroscience letters, 513, 219-222.

Herrera, E., Rodríguez-Ferreiro, J., \& Cuetos, F. (2012). The effect of motion content in action naming by Parkinson's disease patients. Cortex, 48, 900-904. doi:https://doi.org/10.1016/j.cortex.2010.12. 007

Hornykiewicz, O., \& Kish, S. J. (1984). Neurochemical basis of dementia in Parkinson's disease. Journal Canadien des Sciences Neurologiques, 11, 185-190.

Mahon, B. Z., \& Caramazza, A. (2008). A critical look at the embodied cognition hypothesis and a new proposal for grounding conceptual content. Journal of Physiology, 102, 59-70. doi:https://doi.org/10. 1016/j.jphysparis.2008.03.004

Mahon, B. Z., \& Hickok, G. (2016). Arguments about the nature of concepts: Symbols, embodiment, and beyond. Psychonomic Bulletin \& Review, 23, 941-958. doi:https://doi.org/10.3758/ s13423-016-1045-2

Miller Koop, M., Hill, B. C., \& Bronte-Stewart, H. M. (2013). Perceptual errors increase with movement duration and may contribute to hypokinesia in Parkinson's disease. Neuroscience, 243, 1-13. doi: https://doi.org/10.1016/j.neuroscience.2013.03.026

Paivio, A. (1991). Dual coding theory: Retrospect and current status. Canadian Journal of Psychology, 45, 255-287.

Pita-Fernández, S., Pértega-Díaz, S., \& Rodríguez Maseda, E. (2003). La fiabilidad de las mediciones clínicas: El análisis de concordancia para variables numéricas. Cadernos de Atención Primaria, 10 , 290-296.

Rodríguez-Ferreiro, J., Gennari, S. P., Davies, R., \& Cuetos, F. (2011). Neural correlates of abstract verb processing. Journal of Cognitive Neuroscience, 23, 106-118. doi:https://doi.org/10.1162/jocn.2010. 21414

Silveri, M. C., \& Ciccarelli, N. (2007). The deficit for the word-class "verb" in corticobasal degeneration: Linguistic expression of the movement disorder? Neuropsychologia, 45, 2570-2579.

Vigliocco, G., Vinson, D. P., Druks, J., Barber, H., \& Cappa, S. F. (2011). Nouns and verbs in the brain: A review of behavioural, electrophysiological, neuropsychological and imaging studies. Neuroscience \& Biobehavioral Reviews, 35, 407-426. doi:https://doi.org/10.1016/j. neubiorev.2010.04.007

Publisher's note Springer Nature remains neutral with regard to jurisdictional claims in published maps and institutional affiliations. 